\title{
Recent Progress in Distributed Optical Fiber Raman Photon Sensors at China Jiliang University
}

\author{
Zaixuan ZHANG ${ }^{1,2,3}$, Jianfeng WANG ${ }^{1,2,3}$, Yi $\mathrm{LI}^{1,2}$, Huaping GONG ${ }^{1,2}$, \\ Xiangdong YU ${ }^{1,2,3}$, Honglin $\mathrm{LIU}^{1,2,3}$, Yongxing $\mathrm{JIN}^{1,2}$, Juan $\mathrm{KANG}^{1,2}$, Chenxia $\mathrm{LI}^{1,2}$, \\ Wensheng ZHANG ${ }^{2,3}$, Wenping ZHANG $^{2,3}$, Xiaohui NIU ${ }^{2,3}$, Zhongzhou SUN ${ }^{2,3}$, \\ Chunliu $\mathrm{ZHAO}^{1,2}$, Xinyong DONG ${ }^{1,2}$, and Shangzhong $\mathrm{JIN}^{1,2,3}$ \\ ${ }^{1}$ Institute of Optoelectronic Technology, College of Optical \& Electronic Technology, China Jiliang University, \\ Hangzhou, 310018, China \\ ${ }^{2}$ China Jiliang University-BaYang Electric Group United Optical Fiber Sensing Research Center, Hangzhou, 310018, \\ ${ }^{3}$ Hangzhou Optoelectronic Technology Co. Ltd., Hangzhou, 310018, China \\ *Corresponding author: Zaixuan ZHANGＥ-mail: zhangzx@mail.hz.zj.cn
}

\begin{abstract}
A brief review of recent progress in researches, productions and applications of full distributed fiber Raman photon sensors at China Jiliang University (CJLU) is presented. In order to improve the measurement distance, the accuracy, the space resolution, the ability of multi-parameter measurements, and the intelligence of full distributed fiber sensor systems, a new generation fiber sensor technology based on the optical fiber nonlinear scattering fusion principle is proposed. A series of new generation full distributed fiber sensors are investigated and designed, which consist of new generation ultra-long distance full distributed fiber Raman and Rayleigh scattering photon sensors integrated with a fiber Raman amplifier, auto-correction full distributed fiber Raman photon temperature sensors based on Raman correlation dual sources, full distributed fiber Raman photon temperature sensors based on a pulse coding source, full distributed fiber Raman photon temperature sensors using a fiber Raman wavelength shifter, a new type of Brillouin optical time domain analyzers (BOTDAs) integrated with a fiber Raman amplifier for replacing a fiber Brillouin amplifier, full distributed fiber Raman and Brillouin photon sensors integrated with a fiber Raman amplifier, and full distributed fiber Brillouin photon sensors integrated with a fiber Brillouin frequency shifter.

The Internet of things is believed as one of candidates of the next technological revolution, which has driven hundreds of millions of class markets. Sensor networks are important components of the Internet of things. The full distributed optical fiber sensor network (Rayleigh, Raman, and Brillouin scattering) is a $3 \mathrm{~S}$ (smart materials, smart structure, and smart skill) system, which is easy to construct smart fiber sensor networks. The distributed optical fiber sensor can be embedded in the power grids, railways, bridges, tunnels, roads, constructions, water supply systems, dams, oil and gas pipelines and other facilities, and can be integrated with wireless networks.
\end{abstract}

Keywords: Distributed optical fiber sensor, Raman scattering, Rayleigh scattering, Brillouin scattering, fusion principle of optical fiber nonlinear scattering, optical time domain reflectometry (OTDR)

\section{Introduction}

The distributed optical fiber temperature sensor uses optical fiber Raman scattering to measure temperatures, which is an intrinsic type of the optical fiber sensor. In a distributed temperature

Received: 12 September 2011 / Revised version: 30 December 2011

(C) The Author(s) 2012.This article is published with open access at Springerlink.com 
sensor (DTS) system, the optical fiber is not only a transmission medium but also a sensing material. The thermal field along the fiber modulates the intensity of backward Raman scattering (anti-Stokes Raman scattering), and then the backward Raman scattering signal is detected by a photoelectric detector via a wavelength division multiplexer (WDM). After the signal averaging and demodulation, the real-time temperature distribution information will be extracted and presented from noises. Distributed optical fiber temperature sensors can be considered as a kind of the fiber optic temperature measurement network [1]. In the time domain, the time difference of the backscattered light of an injection pulse is proportional to the fiber length that the pulse has traveled, which is often called optical time domain reflectometry (OTDR) principle. So the distributed optical fiber temperature sensor system is also considered as an optical fiber temperature measurement radar system. Distributed optical fiber Raman and Brillouin scattering sensor networks are $3 \mathrm{~S}$ (smart materials, smart structure, and smart skill) systems. They can be embedded and equipped to power grids, railways, bridges, tunnels, roads, constructions, water supply systems, dams, oil and gas pipelines. They can be easily combined with the Internet and wireless networks to constitute Internet of things for identification, display and control.

The research team at China Jiliang University is one of the earliest teams in studying distributed fiber Raman photon sensors. In the past 20 years, we have completed many national, provincial and ministerial research projects, and achieved a number of scientific and technological achievements. Tens of independent intellectual property rights have been industrialized. Some of the achievements and projects are displayed in the following: we cooperated with Shenyang Fire Research Institute of Ministry of Public Security to obtain a support from National "95" Key Scientific and Technological Project, which is named as a new technology of the underground floor for the fire detection and alarm in the large space $[2,3]$; a $6-\mathrm{km}$ distributed optical fiber sensor system using semiconductor lasers (No.D98-A01, 1996) from the Innovation Project of State Bureau of Quality and Technical Supervision. We cooperated with the National Coal Mine Safety Engineering Center to apply a $10-\mathrm{km}$ full distributed fiber Raman photon sensor into the continuous temperature measurement of the mine belt conveyor $[4,5]$, which was awarded the second prize of the Science and Technology Achievements in Zhejiang Province in 2001. We also cooperated with South Korea Electrical Science Research Institute to complete a technology cooperation projects between China and South Korea Ministry of Science and Technology. This project includes the application of a distributed optical fiber temperature measurement system in power industries. We completed a project from the Key Scientific and Technological Project of Zhejiang Province, which was on the application and research of a $30-\mathrm{km}$ distributed optical fiber temperature measurement network (No.011101081, 2000). This work was awarded the second prize of Scientific Research of Higher Schools of Zhejiang Province in 2003 [6, 7]. We also completed a major science and technology project of Zhejiang Province on the development of distributed optical fiber Raman amplifiers (No.021101558,2002), which was awarded the first prize of Scientific Research of Higher School of Zhejiang Province in 2006 [8, 9].

We have published more than two hundred academic articles and have been granted 11 invention patents, 26 utility model patents. In 2006, based on our previous researches in short-range, medium-range, long-range distributed optical fiber Raman temperature sensors and distributed optical fiber Raman amplifiers, we proposed a full distributed optical fiber sensing technology from the fusion theory of optical fiber nonlinear scattering [10]. We have studied and designed a series of full distributed fiber Raman and Brillouin sensors based on the fusion theory of optical fiber nonlinear 
scattering. Many full distributed fiber sensor technologies are proposed, which are new generation ultra-long distance full distributed fiber Raman and Rayleigh scattering photon sensors integrated with a fiber Raman amplifier; ultra-long distance distributed fiber Raman temperature sensors integrated with a fiber Raman amplifier; auto-correction full distributed fiber Raman photon temperature sensors based on Raman correlation dual sources; ultra-long distance full distributed fiber Raman and Rayleigh scattering photon sensors integrated with the fiber Raman frequency shifter; a new type of the Brillouin optical time domain analyzer (BOTDA) with a fiber Raman amplifier, which replaces the fiber Brillouin amplifier with a fiber Brillouin frequency shifter; full distributed fiber Raman and Rayleigh scattering photon sensors and the new generation full distributed fiber Raman and Brillouin photon sensors integrated with a fiber Raman amplifier.

In the past 20 years, the scientific and technological achievements of optical fiber sensing technologies were made into industrialization. A number of distributed optical fiber Raman photon temperature sensors were invented and applied to coal mines, tunnels, automatic fire alarm systems, oil tanks, hazardous environments, the temperature alarm systems for armories, thermal protection and fault diagnosis, hot deformation, stress measurement and the temperature detection and thermal protection of large transformers, generators, dams and the underground cables. In recent years, with the cooperation of Weihai Electric Group, we have established Hangzhou Optoelectronic Technology Co. Ltd., which specializes in the industrial and application engineering. The company already has FGWC series products which include short, medium, and long-range distributed optical fiber Raman temperature sensors, and high precision and high spatial resolution products in the international advanced level.
This paper introduces the recent progress in distributed optical fiber Raman photon sensors based on the optical fiber nonlinear scattering fusion principle at China Jiliang University, especially after getting the support from National Basic Research Program of China (973 Program). Our work is mainly on the optical fiber Raman sensor networks based on the fusion principle of nonlinear optical scattering (No.2010CB327804).

\section{Full distributed fiber optical scattering photon sensor technology based on the fusion principle of optical fiber nonlinear scattering}

There are two kinds of scattering in the interaction between the light and fiber molecules, which are the elastic scattering and the non-elastic scattering. The elastic scattering refers to those whose frequencies of the scattering (or photon energy) remain constant, while the non-elastic scattering refers to those whose frequencies of the scattering (or photon energy) change (increase or decrease). Raman scattering and Brillouin scattering are generated when light waves interact with the media with energy exchanging. When an incident photon is scattered, a low-frequency Stokes or high-frequency anti-Stokes photon is generated, which is resulted from the transitions between vibration levels of the corresponding molecules. Raman scattering includes the Stokes Raman photons which emit optical phonons and the anti-Stokes Raman photons which absorb optical phonons. In optical fibers, the probe light interacts with thermal acoustic waves, which is produced by electrostriction caused by light waves. And the refractive index of the fiber has a periodic modulation (index grating). Thus, the Brillouin photons are generated, which is also called the Brillouin scattering. The Stokes Brillouin photons emit acoustic phonons, and the anti-Stokes Brillouin photons absorb acoustic phonons. 


\subsection{Strain and temperature effects of fiber Brillouin scattering [10, 11]}

Brillouin scattering is an inelastic collision in the interactions between the incident photons and fiber molecules. Because the frequency of acoustic phonons is around $11 \mathrm{GHz}$, the frequency shift of the Brillouin scattering phonon is lower than that of Raman scattering phonon. And the Brillouin peak linewidth is narrower, about $20 \mathrm{MHz}-100 \mathrm{MHz}$. Since the Brillouin gain coefficient is higher, about $5 \times 10^{-11} \mathrm{~m} / \mathrm{W}$, the light intensity of Brillouin scattering is stronger than that of Raman scattering. The frequency shift $v_{\mathrm{B}}$ of backward Brillouin scattering is formulated by

$$
v_{\mathrm{B}}=2 n v / \lambda
$$

where $n$ is the refractive index at the wavelength of incident light $\lambda$, and $v$ is the sound velocity of the optical fiber. For common optical fibers, when $\lambda=1550 \mathrm{~nm}, v_{\mathrm{B}}$ is about $11 \mathrm{GHz}$. The frequency shifts of Brillouin scattering are affected by strains and temperatures:

$$
v_{\mathrm{B}}=v_{\mathrm{B} 0}+\frac{\partial v}{\partial \varepsilon} \varepsilon(\mu \varepsilon)+\frac{\partial v}{\partial T} T\left({ }^{\circ} \mathrm{C}\right) .
$$

So the frequency shift of Brillouin scattering is expressed as

$$
\delta v_{\mathrm{B}}=C_{v \xi} \delta \varepsilon+C_{v T} \delta T
$$

where the strain $\varepsilon$, the strain coefficient $C_{v \xi}$ and the temperature coefficient $C_{v T}$ are

$$
\begin{gathered}
C_{v \varepsilon}=0.0482 \pm 0.004(\mathrm{MHz} / \mu \varepsilon) \\
C_{v T}=1.10 \pm 0.02(\mathrm{MHz} / \mathrm{K}) .
\end{gathered}
$$

Also the intensity rate of Brillouin scattering is affected by strain and temperature:

$$
\frac{100 \delta I_{\mathrm{B}}}{I_{\mathrm{B}}}=C_{P \varepsilon} \delta \varepsilon+C_{P T} \delta T
$$

where the strain coefficient $C_{P \varepsilon}$ and temperature coefficient $C_{P T}$ are

$$
\begin{aligned}
& C_{P \varepsilon}=-(7.7 \pm 1.4) \times 10^{-4} \%(\mu \varepsilon) \\
& C_{P T}=0.36 \pm 0.06 \%(\mathrm{~K})
\end{aligned}
$$

\subsection{Temperature effect of fiber Raman scattering $[3,11]$}

When the incident light interacts with fiber media molecules, the incident light photon emits or absorbs an optical phonon in fiber molecules. The frequency of the optical phonon is $\Delta v=1.32 \times 10^{23} \mathrm{~Hz}$. The Stokes optical photon $h v_{\mathrm{s}}$ and anti-Stokes optical photon $h v_{\mathrm{a}}$ are expressed as

$$
\begin{aligned}
& h v_{\mathrm{s}}=h\left(v_{p}-\Delta v\right) \\
& h v_{\mathrm{a}}=h\left(v_{p}+\Delta v\right)
\end{aligned}
$$

When a laser pulse propagates in an optical fiber, the luminous flux $\phi_{\mathrm{s}}$ of the Stokes backscattering generated by each laser pulse is expressed as

$$
\phi_{\mathrm{s}}=K_{\mathrm{s}} \cdot S \cdot v_{\mathrm{s}}^{4} \cdot \phi_{e} \cdot R_{\mathrm{s}}(T) \cdot \exp \left[-\left(\alpha_{0}+\alpha_{\mathrm{s}}\right) \cdot L\right]
$$

where $L$ is the fiber length. The luminous flux $\phi_{\mathrm{a}}$ of the anti-Stokes backscattering generated by each laser pulse is expressed as

$$
\phi_{a}=K_{a} \cdot S \cdot v_{a}{ }^{4} \cdot \phi_{e} \cdot R_{a}(T) \cdot \exp \left[-\left(\alpha_{0}+\alpha_{a}\right) \cdot L\right]
$$

where $K_{\mathrm{s}}$ and $K_{\mathrm{a}}$ are the coefficients of the Stokes and anti-Stokes scattering across sections, respectively; $v_{\mathrm{s}}$ and $v_{\mathrm{a}}$ are the frequencies of the Stokes and anti-Stokes scattering photons, respectively; $\alpha_{0}, \alpha_{\mathrm{a}}$, and $\alpha_{\mathrm{s}}$ are the average propagation losses of incident, anti-Stokes, and Stokes light in the fiber, respectively; $R_{\mathrm{s}}(T), R_{\mathrm{a}}(T)$ are temperature modulation functions about backward Raman Stokes scattering and backward anti-Stokes scattering:

$$
\begin{gathered}
R_{\mathrm{s}}(T)=[1-\exp (-h \Delta v / k T)]^{-1} \\
R_{\mathrm{a}}(T)=[\exp (h \Delta v / k T)-1]^{-1}
\end{gathered}
$$

The incident photons are scattered by fiber molecules to produce Stokes photons or anti-Stokes photons in the interaction of the laser light and optical fiber molecules, and the transition between vibration levels of the corresponding molecules is achieved. The Stokes Raman photons emit optical phonons, and the anti-Stokes Raman photons absorb optical phonons. The intensity rate $I(T)$ of the Stokes and anti-Stokes Raman backscattering is expressed as

$$
I(T)=\frac{\phi_{\mathrm{a}}}{\varphi_{\mathrm{s}}}=\left[\frac{v_{\mathrm{a}}}{v_{\mathrm{s}}}\right]^{4} \mathrm{e}^{-\left(\frac{h \Delta v}{k T}\right)}
$$

where $h$ is the Planck's constant, $h=$ $6.6260687652 \times 10^{-34} \mathrm{~J} \cdot \mathrm{s}, \Delta v$ is the frequency 
(about $13.2 \mathrm{THz}$ ) of the optical phonon of the fiber molecules, $k$ is the Boltzmann constant, $k=1.380650324 \times 10^{-23} \mathrm{~J} \cdot \mathrm{K}^{-1}$, and $T$ is the Kelvin's absolute temperature. The temperature information of each fiber section can be obtained by the intensity rate of the Stokes and anti-Stokes Raman backscattering.

During the temperature of the calibration fiber $T=T_{0}$, the relation between the Raman intensity rate and temperature is

$$
\frac{I_{\mathrm{a}}(T) / I_{\mathrm{s}}(T)}{I_{\mathrm{a}}\left(T_{0}\right) / I_{\mathrm{s}}\left(T_{0}\right)}=\frac{\mathrm{e}^{-h \Delta v / k T}}{\mathrm{e}^{-h \Delta v / k T_{0}}} .
$$

We can obtain from the expression above

$$
\frac{1}{T}=\frac{1}{T_{0}}-\frac{k}{h \Delta v} \ln \frac{\phi_{\mathrm{a}}(T) / \phi_{\mathrm{s}}(T)}{\phi_{\mathrm{a}}\left(T_{0}\right) / \phi_{\mathrm{s}}\left(T_{0}\right)} .
$$

In actual measurements, $\phi_{\mathrm{a}}(T), \phi_{s}(T)$, $\phi_{a}\left(T_{0}\right), \phi_{s}\left(T_{0}\right)$ can be obtained, and the temperature $T$ can be calculated by the formula above.

\subsection{Amplification effect of Stimulated Raman scattering [10, 12-19]}

During the intensity of incident light exceeds the Raman threshold power of the fiber, the power of Stokes Raman scattering rapidly increases in the fiber media, and most of the pumping power is converted into the Stokes Raman scattering power. This phenomenon is called stimulated Raman scattering. The initial growth of the Raman scattering power can be expressed by the following formula:

$$
\frac{d I_{\mathrm{s}}}{d z}=g_{\mathrm{R}} I_{\mathrm{p}} I_{\mathrm{s}}
$$

where $I_{\mathrm{s}}$ is the intensity of the Stokes waves, $I_{\mathrm{p}}$ is the intensity of the pumping light, and $g_{R}$ is the coefficient of the Raman gain. The most prominent characteristic of the Raman gain in optical fibers is that $g_{\mathrm{R}}$ has a very broad frequency range (about $40 \mathrm{THz}$ ) and has a very large peak linewidth near the 13.2 THz. The gain of the fiber Raman amplifier is given as

$$
G_{A}=\frac{I_{\mathrm{s}}(L)}{I_{\mathrm{s}}(0) \exp \left(-\alpha_{\mathrm{s}} L\right)}=\exp \left(g_{\mathrm{R}} P_{0} L_{\mathrm{eff}} / A_{\mathrm{eff}}\right)
$$

where $P_{0}=I_{0} A_{\text {eff }}$ is the input pumping power of the amplifier, $L_{\text {eff }}$ is the effective length of the fiber, $A_{\text {eff }}$ is the effective cross section of the fiber, $g_{\mathrm{R}}$ is the Raman gain coefficient in optical fibers, and $\omega$ is the operation frequency. The effective bandwidth of the fiber Raman amplifier (FRA) is expressed as

$$
B_{\text {eff }}=\left(\frac{2 \pi}{I_{0} L_{\text {eff }}}\right)^{1 / 2}\left|\frac{\partial^{2} g_{\mathrm{R}}}{\partial \omega^{2}}\right|_{\omega=\omega_{\mathrm{s}}}^{-1 / 2}
$$

The threshold condition of the FRA could be expressed as

$$
P_{\mathrm{s} 0}^{\text {eff }} \exp \left(g_{\mathrm{R}} P_{0} L_{\text {eff }} / A_{\text {eff }}\right)=P_{0} .
$$

We can see from (12) that $P_{\mathrm{s} 0}^{\text {eff }}$ is also related to $P_{0}$.

In an FRA, the noise mainly comes from the beat noise between the spontaneous emission and the signal beam, as well as the shot noise that comes from the signal beam. So the noise-factor of the FRA can be expressed as

$$
N F=P_{\mathrm{ASE}} /\left(h v B_{0} G\right)+1 / G
$$

where $P_{\mathrm{ASE}}$ is the power of the amplification spontaneous emission (ASE), $B_{0}$ is the frequency resolution of the ASE power measurement, and $G$ is the on-off gain of the FRA.

\subsection{Amplification effect of Rayleigh and Brillouin scattering in the fiber Raman amplifier [19-22]}

The amplification effect of Rayleigh and the Brillouin back scattering in the distributed optical Raman amplifiers was studied. The amplification effect of Rayleigh and Brillouin scattering in the distributed fiber Raman amplifiers with the backward pumping was researched. Figure 1 shows the experimental setup, which consists of a detection laser, two isolators, a bidirectional coupler, a single-mode fiber, a pumping-signal coupler, and an optical spectrum analyzer (OSA). The detection laser consists of four tunable external-cavity lasers, in the region of $1500 \mathrm{~nm}-1600 \mathrm{~nm}$. The laser bandwidth is less than $10 \mathrm{MHz}$, its output power can be tuned from $-7 \mathrm{dBm}$ to $-3 \mathrm{dBm}$, and its signal-to-noise ratio (SNR) is $54 \mathrm{~dB}$. The single mode fiber is $25-\mathrm{km}$-length $\mathrm{G} 652$ fiber. A $1427-/ 1520-\mathrm{nm}$ coarse wave division multiplier 
(CWDM) is used as the pumping-signal coupler. A Raman fiber laser is used as the pumping laser, working at the wavelength of $1427.2 \mathrm{~nm}$. The spectral linewidth of the laser is $0.067 \mathrm{~nm}$ with the output power in the region of $0-1200 \mathrm{~mW}$.

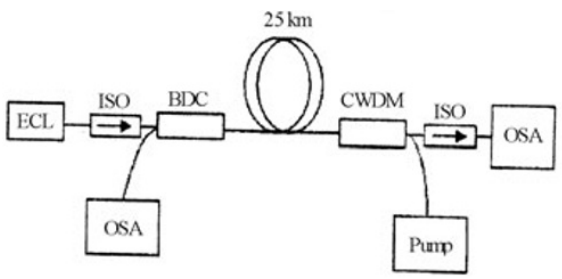

Fig. 1 Experiment setup to verify the amplification effect of Rayleigh scattering.

The second Stokes Brillouin scattering, first backward anti-Stokes Brillouin scattering, and backward Rayleigh scattering were observed when an 800-mW pumping was used in the distributed fiber Raman amplifier. Figure 2 shows the second order Stokes Brillouin scattering, the first order anti-Stokes Brillouin scattering $(1521.21 \mathrm{~nm}$ or 197.2109 THz), and the backward Rayleigh scattering $(1521.13 \mathrm{~nm})$.

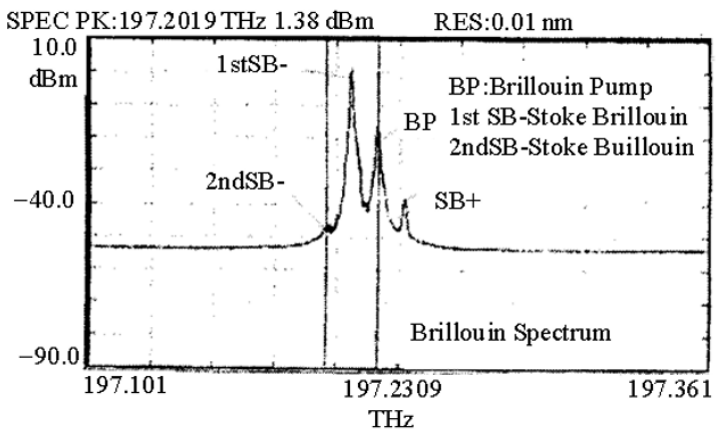

Fig. 2 Backward Rayleigh and Brillouin scattering in the FRA.

Figure 3 shows that the intensities of backward Stokes Brillouin scattering, anti-Stokes Brillouin scattering, and Rayleigh scattering change with the pump power. Figure 4 shows the curves of the gain variation of backward Stokes Brillouin and anti-Stokes Brillouin scattering.

From Fig. 4, we can see that the saturation gain of Rayleigh scattering is $23 \mathrm{~dB}$, and the saturation gain of Brillouin Stokes scattering is $50 \mathrm{~dB}$ when the pump power of the FRA is $800 \mathrm{~mW}$.

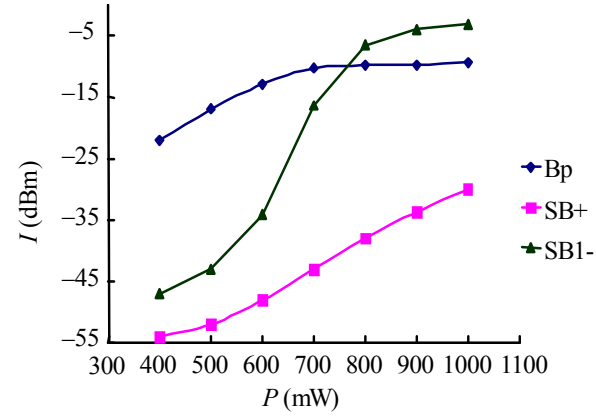

Fig. 3 Intensities of Rayleigh and Brillouin scattering vs pump powers.

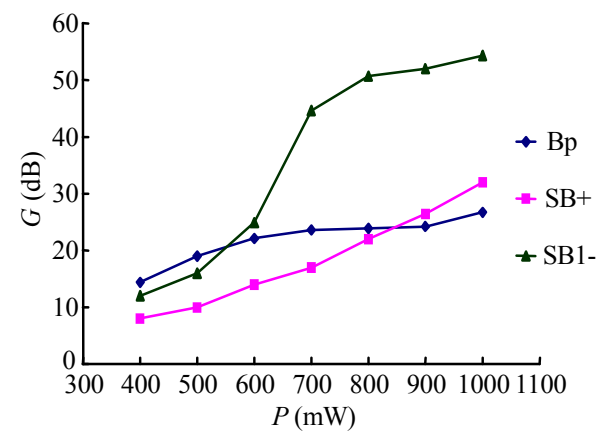

Fig. 4 Gains of Rayleigh and Brillouin scattering vs pump powers.

\subsection{Fiber sensor technology based on the fusion principle of fiber nonlinear scattering [22]}

The spectra of Rayleigh scattering and nonlinear Raman and Brillouin scattering in the fiber are shown in Fig. 5. There co-exist many kinds of scattering in fibers, whose spectral characteristics are different, as shown in Table 1.

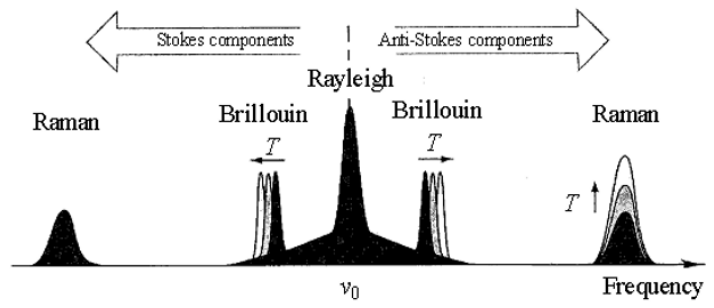

Fig. 5 Spectra of Rayleigh, Brillouin and Raman scattering in fibers.

The Raman scattering is an inelastic collision in the interactions between the incident photon and fiber molecules. Because the frequency of optical phonons is $13.2 \mathrm{THz}$, the frequency shift of the Raman scattering photons is quite large. The Brillouin scattering is also an inelastic collision in the interactions between the incident photon and 
fiber molecules. Because the frequency of acoustic phonons is around $11 \mathrm{GHz}$, the frequency shift of the Brillouin scattering photons is smaller compared to the Raman scattering. Besides, the most predominant characteristic of the Raman gain is its broad frequency range $(40 \mathrm{THz})$ and large peak linewidth (about $5 \mathrm{THz}$ ) in the center of the frequency shift. The coefficient of the Raman gain is small (about $7 \times 10^{-14} \mathrm{~m} / \mathrm{W}$ ), and the stimulated threshold power is high. However, the most dominant characteristic of the Brillouin gain is its narrow frequency range, only about $20 \mathrm{MHz}-$ $100 \mathrm{MHz}$. The Brillouin gain coefficient is around $7 \times 10^{-11} \mathrm{~m} / \mathrm{W}$, which is larger by three orders of magnitude than the Raman gain coefficient. Also, the stimulated threshold power is lower than that of stimulated Raman scattering. The main characters of spontaneous anti-Stokes Raman scattering and Brillouin scattering at the wavelength of $1550 \mathrm{~nm}$ are shown in Table 1.

Table 1 Physical performances of fiber nonlinear optical scattering.

\begin{tabular}{lll}
\hline \multicolumn{1}{c}{ Parameter } & Raman & \multicolumn{1}{c}{ Brillouin } \\
\hline Frequency shift $(\mathrm{GHz})$ & $13.2 \mathrm{k}$ & 11 \\
Band width $(\mathrm{MHz})$ & $\sim 5 \mathrm{M}$ & $\sim 20-100$ \\
Gain coefficient $10^{-11}(\mathrm{~m} / \mathrm{W})$ & $\sim 7 \times 10^{-3} \sim 5$ \\
Scattering power ratio (Rayleigh) $(\mathrm{dB})$ & $\sim 30$ & $\sim 15$ \\
Temperature sensitivity $\left({ }^{\circ} \mathrm{C}\right)$ & $\sim 0.8 \%$ & $\sim 0.3 \%$ \\
Frequency shift temperature sensitivity $\left(\mathrm{MHz} /{ }^{\circ} \mathrm{C}\right)$ & - & 1.1 \\
Intensity strain sensitivity $(\mu \varepsilon)$ & - & $-9 \times 10^{-4} \%$ \\
Strain sensitivity $(\mathrm{MHz} / \mu \varepsilon)$ & - & 0.048 \\
\hline
\end{tabular}

The principles of the full distributed fiber sensors are established on fiber nonlinear scattering using the physical properties of a number of linear and nonlinear scattering effects. The main characteristics include:

(1) The Brillouin scattering and the stimulated Brillouin scattering of the optical fiber have strain and temperature effects, which can be used as a distributed temperature and strain fiber sensor. The stimulated Brillouin scattering of the optical fiber has an optical amplification effect and can be used as a narrow band optical fiber Brillouin amplifier. The spontaneous Raman scattering of the optical fiber has temperature effect and can be used as a distributed optical fiber temperature sensor. The stimulated Raman scattering of the optical fiber has an optical amplifier effect and can be used as a broad band optical fiber Raman amplifier.

By utilizing the physical characteristics of these scattering effects, the multi-function optical fiber devices have been made based on the hybrid nonlinear scattering effects. The long-distance distributed optical fiber sensor with multi-parameters and high performance has been proposed based on its different physical characteristics.

(2) The frequency shifts of Raman scattering and Brillouin scattering in the optical fiber have a difference of $10^{3}$ orders in quantity. So Raman scattering and Brillouin scattering have different frequencies even using the same probing laser. Utilizing the principle of the WDM, the cross interference will not be induced. So the distributed Raman and Brillouin scattering photonic sensors have been used.

(3) The bandwidths of Raman scattering and Brillouin scattering in optical fibers have a difference of $10^{4}$ orders in quantity. According to the principle of the wave division multiplier, adopting two probing lasers with different wavelengths and different bandwidths, based on the principle of hybrid nonlinear scattering effects, the multi-function distributed Raman and Brillouin scattering photonic sensor can be realized.

(4) According to the principle of the wave division multiplier, adopting probing lasers and pumping lasers with different wavelengths and different bandwidths, based on the hybrid effects of spontaneous scattering and stimulated scattering in the fiber, the spontaneous Raman scattering and the spontaneous Brillouin scattering can be amplified in the distributed fiber Raman amplifier. The long-distance multi-function distributed Raman and Brillouin scattering photonic sensor combined with 
the Raman scattering can be realized.

(5) The powers of the probing laser and the pumping laser must be carefully controlled to prevent the production of cascaded stimulated Brillouin scattering and the interference of four-wave mixing and others nonlinear effects in fibers.

By utilizing the physical characteristics of these light scattering effects, the multi-function optical fiber devices can be realized based on the hybrid nonlinear scattering effects. Professor Z. Zhang and his team at China Jiliang University (CJLU) have researched and designed a series of full distributed Brillouin and Raman fiber sensors based on the hybrid nonlinear scattering effects. We have published more than two hundred academic articles and has been granted 11 invention patents, 26 utility model patents, which are ultra-long distance full distributed fiber Raman and Rayleigh sensors combined with the FRA, remote distributed Raman temperature fiber sensors combined with the FRA, a new generation of the fiber Brillouin optical time domain analyzer combined with the Raman fiber amplifier, an auto-correction distributed optical fiber Raman temperature sensor with the two-wavelength Raman laser, an ultra-long distance full distributed fiber sensor combined with the optical fiber Raman frequency shift device, a distributed optical fiber Raman photon temperature sensor for the fire detection, a distributed fiber Raman and Rayleigh scattering photonic sensor, a distributed fiber Brillouin sensor combined with the fiber Brillouin frequency shift device, and a new generation of the distributed fiber Raman and Brillouin scattering photonic sensor.

\section{Full distributed fiber Raman photon sensors}

\subsection{Full distributed fiber photon sensors based on Raman and Rayleigh scattering integrated with a fiber Raman amplifier [23-25]}

In 2007, Z. Zhang team [24] at China Jiliang University proposed an extended range of the distributed fiber Raman photonic temperature sensor system integrated with a Raman amplifier. The system was designed according to the intrinsic characteristics of optical fibers, the amplification effect of the stimulated Raman scattering theory in optical fibers, the anti-Stokes Raman scattering wave strength of the fiber optic temperature modulation, and the OTDR principles. It was based on the hybrid theory of the stimulated Raman scattering and the anti-Stokes Raman scattering of optical fibers and the wave division multiplexing principle. The sensor included the pulsed semiconductor lasers, the pumped-signal optical wavelength division multiplexer, and the pumped fiber laser connected with a $1 \times 2$ optical fiber bi-directional coupler whose one end was connected with the 50-km-length optical fiber. By measuring the intensities of the backward Rayleigh scattering wave, the Stokes and anti-Stokes Raman scattering waves respectively, the temperature and strain information of the optical fiber can be obtained. The hybrid distributed fiber Raman amplifier and distributed fiber Raman photon temperature sensor were incorporated into a new system, which had been granted as a national utility model patent. It improved the signal-to-noise ratio of the system and the capacity range of the distributed fiber Raman temperature sensor. The experimental setup is shown in Fig. 6.

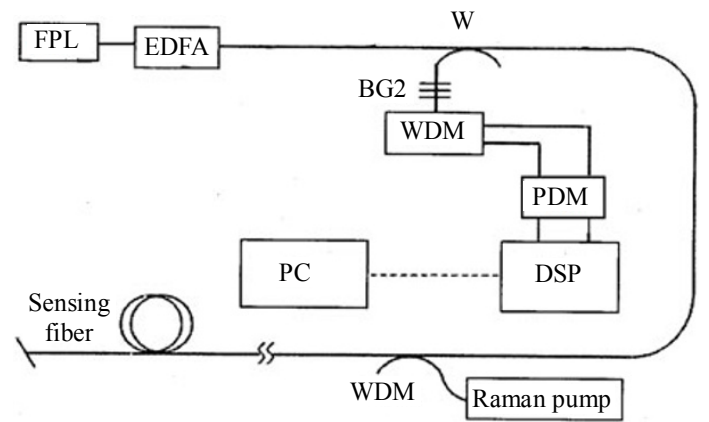

Fig. 6 Experimental setup of the distributed fiber Raman photon sensor integrated with a fiber Raman amplifier.

\subsection{Full distributed fiber Raman photon sensors based on Raman correlation sources [26-28]}

The Raman frequency shift is $13.2 \mathrm{THz}$. So the 
wavelength difference of the anti-Stokes Raman scattering light and the Stokes Raman scattering light is much larger. Because of the chromatic dispersion of the fiber, the spreading speeds of the back anti-Stokes Raman scattering light and the Stokes Raman scattering light in the fiber are different. This "difference step" phenomenon in the time domain reflection curve of the backward anti-Stokes Raman scattering light and backward Stokes Raman scattering light of the fiber is generated. In distributed fiber Raman temperature sensors, we usually use the backward Stokes Raman scattering light of the time domain signal to demodulate the back anti-Stokes Raman scattering light of the time domain signal of the fiber and to get the temperature information of the fiber. The "difference step" phenomenon reduces the spatial resolution and the temperature measurement precision of the system. Since the fiber loss of different wavelength regions is different, there are spectral effects of the fiber loss. When using the Stokes Raman reference channel to demodulate the anti-Stokes Raman signal in the temperature measurement, the temperature demodulation curve becomes tilted and aberrant. Then it causes temperature measurement errors and reduces temperature measurement precision. On the other hand, bending, compressing and stretching of the fibers are easy to cause fiber nonlinear phenomena and result in the difference of the loss of each wavelength region, and the location of the optical cable is random. It is difficult to adjust artificially, so it needs to adopt the auto-correction method.

In 2009, Z. Zhang team [27] at China Jiliang University proposed a new method using a signal acquisition, processing system and an auto-correction temperature calibrating fiber, to achieve the auto-correction of dispersion and loss spectra and to solve auto-correction of the light source. This method improved the stability of the system. The auto-correction distributed optical fiber Raman temperature sensor includes Raman correlation dual wavelength sources, a WDM, (wave division multiplier), a photoelectric receiving module, a digital signal processor (DSP), and a computer. The Raman correlation dual wavelength sources contain a main laser and an assistant laser, and their wavelength difference is Raman correlation wavelength. The intensity ratio between the backward anti-Stokes Raman scattering and the backward Stokes Raman scattering is shown as

$$
R(T)=\frac{I_{1, \mathrm{as}}}{I_{2, \mathrm{~s}}}=\frac{I_{1}}{I_{2}}\left(\frac{\lambda_{2, \mathrm{~s}}}{\lambda_{1, \mathrm{as}}}\right) \exp \left(-\frac{h c v}{k T}\right) \frac{\varphi\left(\lambda_{1}, l\right) \varphi\left(\lambda_{1, \mathrm{ss}} l\right)}{\varphi\left(\lambda_{2}, l\right) \varphi\left(\lambda_{2, \mathrm{~s}} l\right)}
$$

where $\quad \lambda_{2, \mathrm{~s}}=\lambda_{1}, \lambda_{1, \mathrm{as}}=\lambda_{2} \quad, \quad \varphi\left(\lambda_{1, \mathrm{as}} l\right)=\varphi\left(\lambda_{2}, l\right) \quad$, $\varphi\left(\lambda_{2, s} l\right)=\varphi\left(\lambda_{1}, l\right)$.

Then we can get

$$
R(T)=\frac{I_{1}}{I_{2}}\left(\frac{\lambda_{1}}{\lambda_{2}}\right)^{4} \exp \left(-\frac{h c v}{k T}\right)
$$

In this expression, the wavelength $\lambda_{1}$ of the main laser is $1550 \mathrm{~nm}$, and the wavelength $\lambda_{2}$ of the assistant laser is $1450 \mathrm{~nm}$.

The temperature of the calibration optical fiber is $T_{0}$, and the temperature $T$ of the sensor fiber can be expressed as

$$
T=\left[\frac{1}{T_{0}}-\frac{k}{h c v} \ln \left(\frac{R(T)}{R\left(T_{0}\right)}\right)\right] .
$$

The experimental setup is shown in Fig. 7.

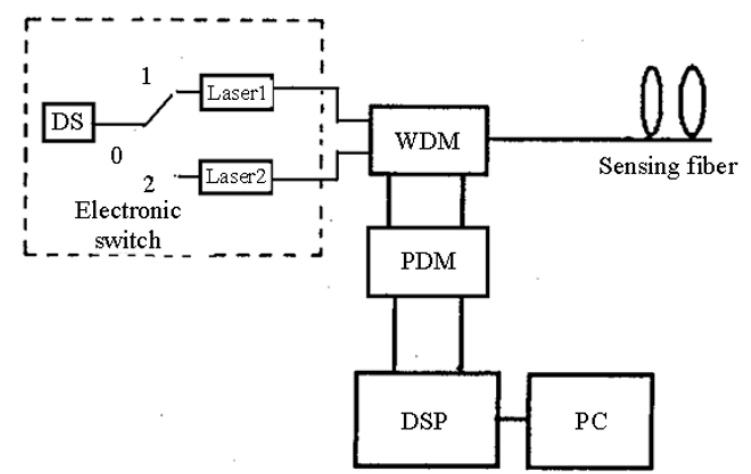

Fig. 7 Experimental setup of the auto-correction distributed fiber Raman sensor (DS: drive source; PDM: photoelectric diode module).

\subsection{Full distributed fiber Raman photon sensors based on pulse encoding modulated sources [29]}

In distributed optical fiber sensors, in order to 
improve the spatial resolution of the system, it is necessary to compress the pulse width of the laser. In order to improve the signal-to-noise ratio of the system, it needs to increase the power of the laser. But when the power of the incident light exceeds the threshold power of the fiber, nonlinear phenomena will occur, and the system will be in an unstable state.

In recent years, for distributed optical fiber sensors (Raman, Brillion), we usually use the pulse coding modulation light source, i.e. laser sequence pulses encoding technology. It uses the laser sequence pulse as a launch source to improve the number of launch photons. On the foundation of not affecting the spatial resolution of the system, the backward signal strength of the fiber can be improved greatly; thereby, the signal-to-noise ratio of the system is improved, and the nonlinear effect of the fiber is repressed effectively. Because of using encoding and decoding technologies, it improves the ability of the extraction and distinction of the system's signal. Using the same signal averaging time, we can obtain a better signal-to-noise ratio. The improvement of the signal-to-noise ratio obtained by $N$ bits of the sequence pulse is determined by (23). The spatial resolution of the distributed fiber sensor system with a pulse coding modulation light source is determined by the composition sequence pulses of the minimum pulse width of the encode pulse and the bandwidth of the receiver.

$$
S N R_{N}=\sqrt{\frac{\sigma^{2}}{N}} / \sqrt{\frac{\sigma^{2}}{(N+1)^{2}}}=\frac{N+1}{2 \sqrt{N}} .
$$

We can learn from (23) that signal-to-noise ratio can be improved with the improvement of the code number $N$, zero-mean random process with variance $\sigma^{2}$. When $N$ is equal to 255 , we can get

$$
S N R_{255}=\frac{255+1}{2 \sqrt{255}} \approx 8.02 \text {. }
$$

In 2010, the China invention patent named "distributed optical fiber Raman temperature sensor using serious pulse code and decode optical source" was granted to $\mathrm{X}$. Yu team at China Jiliang University [29]. The distributed optical fiber temperature measurement system, using the pulse sequence encoding and decoding, based on the $s$-matrix transformations to encode and decode the signal, uses the effect that the intensity of the optical fiber Raman light is modulated by the temperature and optical time domain reflection principle to conduct optical fiber online positioning of the temperature measurement. The sensor includes fiber pulsed coding laser transmitters, an integrated-optic WDM, fiber temperature sampling loops, sensing fibers, two photoelectric receivers, two amplifier modules (PDM1, PDM2), a DSP for encoding and decoding demodulation, and a computer, shown in Fig. 8 .

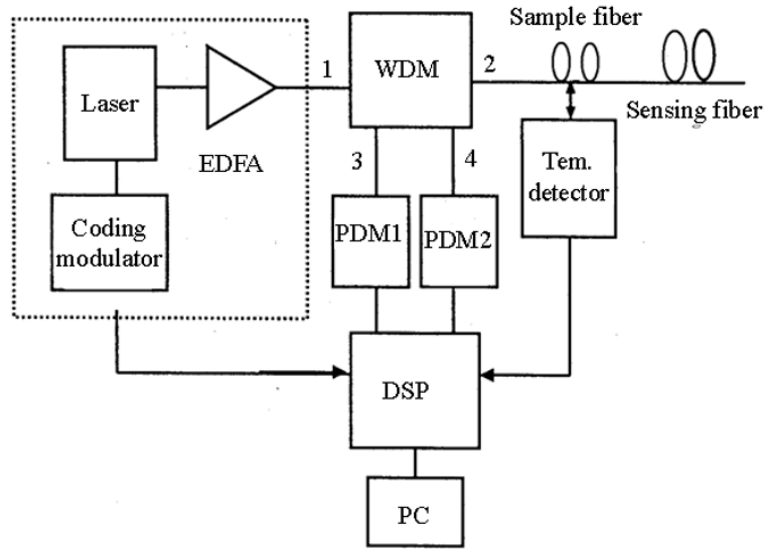

Fig. 8 Experimental setup of the distributed fiber Raman photon sensor using pulse code modulated sources.

The distributed optical fiber temperature measurement system uses pulse encoding and decoding, based on the $s$-matrix transformations to encode and decode the signal. The sensor uses the novel sequence number of the laser pulse encoding and decoding technology under the same measurement time, and this sensor can get a better signal-to-noise ratio. Meanwhile, this sensor increases the number of transmitting photons and improves spatial resolution by narrowing the pulse width of the laser. The technology can be applied to the distributed optical fiber temperature sensing 
system with an extended range and high spatial resolution.

\subsection{Full distributed fiber Raman photon sensors incorporating the fiber Raman frequency shifter $[30,31]$}

Ultra-long range distributed optical fiber Rayleigh and Raman scattering sensors incorporating an optical fiber Raman frequency shifter is based on the principle of the optical fiber Raman frequency shift and the fiber optical sensing for the fusion of Rayleigh and the Raman scattering. The sensor consists of a pulse coding laser, a fiber Raman frequency shifter (Raman shifter), an integrated-optic WDM, and the OTDR includes a fiber pulse laser, a single mode fiber and a $1660-\mathrm{nm}$ band-pass filter composed of a Raman frequency shifter, an integrated-optic WDM, sensing fibers, a photoelectric diode module (PDM), DSP and computers. The sensors are of low-cost, long-life, and simple structure and have high SNRs, good reliability, and extended range from $15 \mathrm{~km}$ to $60 \mathrm{~km}$, which can be applied to the petrochemical pipelines, tunnels, large civil engineering monitoring and disaster monitoring.

When the incident laser at the frequency of $v_{0}$ and the optical fiber have produced nonlinear interaction, the incident laser photon may be absorbed or release a phonon, called anti-Stokes Raman scattering photon or Stokes Raman scattering photon.

$$
v=v_{0} \pm \Delta v
$$

where $\Delta v$, the phonon frequency of the fiber, is 13.2 THz, making incident laser has a frequency shift. It is called the fiber Raman frequency shift, according to which we can make a Raman frequency shifter. A pulse laser has the center wavelength of $1550 \mathrm{~nm}$ and the spectrum width of $0.1 \mathrm{~nm}$. The width of the laser pulse is $10 \mathrm{~ns}$ with the peak power of $1 \mathrm{~W}-1 \mathrm{~kW}$ that can be adjusted, and its repeated frequency is $500 \mathrm{~Hz}-20 \mathrm{kHz}$. Because different fiber lengths have different conversion efficiencies, the best length of the fiber of the Raman frequency shifter is $1 \mathrm{~km}$.

The peak power of the $1660-\mathrm{nm}$ broadband Stokes Raman shifter increases with an increase in the pump power of the fiber. When the pump power reaches or surpasses the threshold power of the fiber stimulated Raman scattering, the intensity of the Raman shifter increases significantly. At this time, most of the pump power can be converted to the Stokes Raman light and result in the Raman amplification phenomenon. Along with an increase in the pump power, the saturation phenomenon of the peak power of the Raman shifter will emerge. If the pump power continues to increase, the second-order Raman phenomenon will appear. The spectra of the optical fiber Raman frequency shifter device in different pump powers are shown in Fig. 9. Full distributed fiber Raman photons sensors fused on the fiber Raman frequency shifter is shown in Fig. 10.

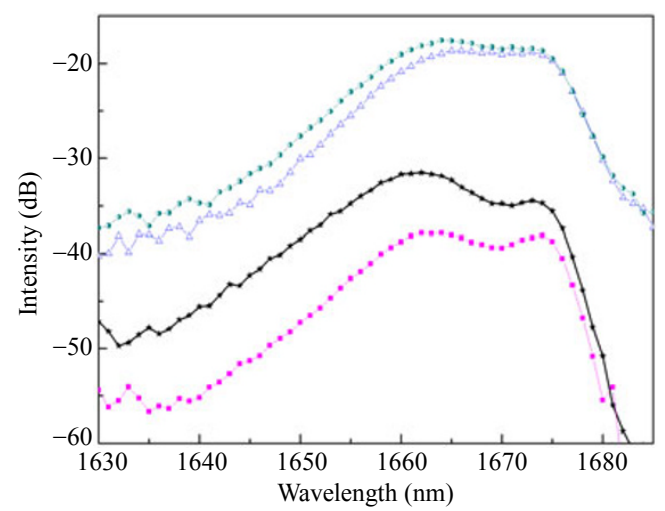

Fig. 9 Spectra of a fiber Raman shifter.

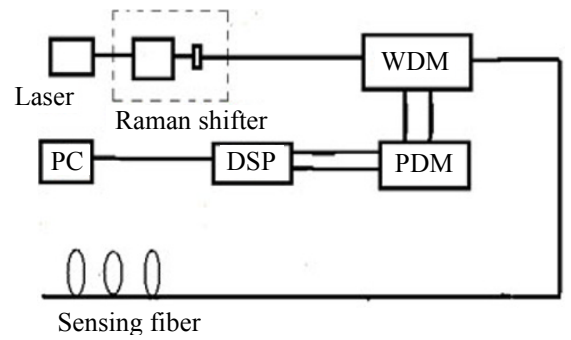

Fig. 10 Full distributed fiber Raman photon sensors fused with a fiber Raman frequency shifter.

The fiber Raman frequency shifter is suitable for fiber sensor lights, whose spectral bandwidth can 
reach $28 \mathrm{~nm}$ and gain can suppress optical fiber transmission loss, improving the signal to noise ratio of the distributed optical fiber temperature sensor system and the spatial resolution of the sensor, thus increasing the sensing distance.

An ultra-long range full distributed optical fiber Rayleigh and Raman scattering sensor incorporating optical fiber Raman frequency shifter includes a high power fiber pulse laser, a Raman shifter composed of single mode fibers and a 1660-nm band-pass filter, an integrated-optic WDM, sensing fibers, a PDM, a DSP, and a computer.

\section{Distributed optical fiber Raman and Brillouin scattering photon sensors}

\subsection{New generation of the BOTDA integrated with the fiber Raman amplifier [32]}

The BOTDR uses the spontaneous Brillouin scattering in fibers, and the intensity of the backward Brillouin scattering signal is very weak. So the resolution is very low, and the sensing range is limited if using the frequency shift and the intensity ratio of the initiative and metrical backward Brillouin scattering signal. T. Horiguchi proposed a type of the BOTDA, which added a coherent pump in the end of the fiber, in order to realize a Brillouin amplifier. The coherent stimulated Brillouin scattering enhances the intensity of the signal and improves the signal to noise ratio. But the BOTDA system needs to lock the frequency difference of the probing laser and the pumping laser strictly, and it is technically difficult to control.

In 2008, Z. Zhang team proposed a new BOTDA system, which was based on the broadband nonlinear amplifying effect, temperature and strain effects of Brillouin scattering, and the principle of the optical time domain analyzer. The experimental setup is shown in Fig. 11, which contains a narrow linewidth single frequency fiber laser, a fiber splitter, a pulse modulator, two fiber circulators, a heterodyne receiver, a DSP, a fiber grating filter, single mode fibers, and a Raman pump laser. In the experiment, a high power Raman laser was used as the pump laser, which avoided the difficulty of locking the frequency difference of the probing laser and the pumping laser strictly, and the broadband fiber nonlinear scattering amplifier was utilized to replace the narrow-band fiber Brillouin amplifier. Therefore, the gain of the backward Brillouin scattering signal increased. The signal to noise ratio was improved, and the sensing length could reach $30 \mathrm{~km}$. The strain resolution and temperature resolution were also improved simultaneously.

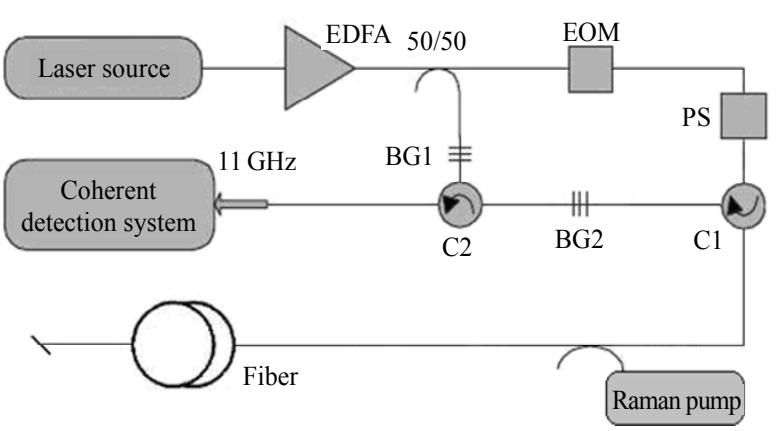

Fig. 11 New generation of Brillouin time domain analyzers.

A tunable narrow band semiconductor external cavity laser (ECL) is used as the signal source of stimulated Brillouin scattering (SBS). Its wavelength is $1550 \mathrm{~nm}$ and linewidth is less than $10 \mathrm{MHz}$. The erbium doped fiber amplifier (EDFA) is used to amplify the continue wave (CW) laser of the ECL. The amplified light passes through the electro-optical modulator (EOM) to generate the laser pulse with the narrow line width and high power by using a 50:50 optical fiber coupler, which is used as the SBS pump of $1550 \mathrm{~nm}$. Then the polarization correlation of the laser pulse is reduced by a polarization scrambler, the laser pulse is injected into the sensing fiber through the optical fiber circulator $\mathrm{C} 1$ through the coupler acting as the reference light of the coherent detection for the Brillouin signal, and passes through the fiber circulator $\mathrm{C} 2$ after a fiber Bragg grating BG1. The Rayleigh scattering light of the backscattering signal 
is filtered by a narrow line width fiber Bragg grating BG2 through $\mathrm{C} 1$, and then the rest backscattering light passes through C2. Finally, the Brillouin backscattering signal can be obtained at the position of BG1, which enters into the coherent detection system of the Brillouin scattering signal through $\mathrm{C} 2$, and the strain information is obtained by the optical demodulation of the probe signal and the Brillouin frequency demodulation. The Raman pump is employed to amplify the Brillouin scattering signal. The ultra-long distance strain sensing of the Brillouin signal using the effect of the Raman amplifier to avoid the power loss caused by long distance transmission can be realized. The noise suppression of this system mainly depends on BG1 and BG2. BG1 with a reflection bandwidth of $0.1 \mathrm{~nm}$ only reflects the Stokes Brillouin scattering signal around $1550 \mathrm{~nm}$, and BG2 can further control the Rayleigh scattering signal caused by the Raman pump laser operating at $1465 \mathrm{~nm}$. The WDM also restrains the noise effectively. The receiving part detector improves the signal to noise ratio, picks up the useful signals by utilizing cumulative averaging and low-pass filtering, and meanwhile matches up with BOXCAR sample integration.

\subsection{New generation of the ultra-long distance full distributed fiber Raman and Brillouin scattering photon sensor [33-35]}

In 2007, Z. Zhang team proposed an ultra-long distance distributed fiber Raman and Brillouin photonic sensor combined with a fiber Raman amplifier, which was based on the temperature sensitive effect of Raman scattering, the strain and temperature sensitive effects of Brillouin scattering, the amplifying effect of stimulated Raman scattering, and the optical time domain reflection principle. The sensor system was based on the hybrid principle of the spontaneous Raman scattering, the stimulated Raman scattering, and the stimulated Brillouin scattering. It combined the distributed fiber Raman temperature sensor, the distributed fiber Brillouin sensor, and the distributed fiber Raman amplifier and then formed a new ultra-long distance distributed fiber temperature and strain sensor. The temperature information was obtained by detecting the intensity ratio between the anti-Stokes Raman scattering and the Stokes Raman scattering, which were injected into the detecting system through two band pass filters. The strain distribution information along the fiber was acquired by detecting the Brillouin frequency shift, which was caused by coherently detecting the backward Brillouin scattering signal through a fiber grating filter and the local narrow-band oscillation laser. By combining the fiber Raman amplifier, the signal to noise ratio could be improved. Especially, the sensor system used two probing light sources: one was a broadband Fabry-Perot (F-P) laser diode operating at $1550 \mathrm{~nm}$, which was used as the probing laser of the fiber Raman scattering sensor; the other was a 1555-nm narrow band external cavity ECL laser, which acted as the probing laser of the fiber Brillouin scattering sensor. The pump source of the fiber Raman amplifier was a high power tunable fiber Raman laser with the central wavelength of $1465 \mathrm{~nm}$. The single mode sensing fiber was $100 \mathrm{~km}$ long, and the temperature resolution was $\pm 2{ }^{\circ} \mathrm{C}$. The experimental setup is shown in Fig. 12. This work has been awarded State Intellectual Property Office Authorization [34].

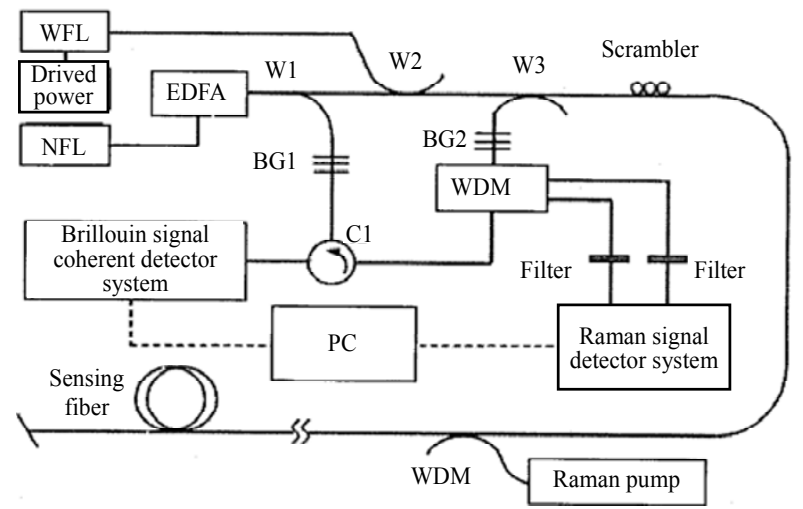

Fig. 12 New generation of the ultra-long distance distributed optical fiber Raman, Brillouin sensor combined with the FRA. 
The light of the wide bandwidth fiber laser (WFL) is amplified by an EDFA and injected into the ultra-long distance single mode sensing fiber through a polarization scrambler and a WDM. The backscattering signal in return passes the fiber splitter, a narrow bandwidth reflection filter and the WDM which restrains the Rayleigh scattering caused by the 1465-m Raman pump laser. The backward signal passes through the Raman signal detecting system which is used to receive and amplify the photo-electricity, and then it is injected into the PC control center for demodulation. The position information of temperatures and strains along the sensing fiber is thus obtained. The operation wavelength of the Raman pump laser is $1551.83 \mathrm{~nm}$. The spectral bandwidth is $0.067 \mathrm{~nm}$, and the power can be tuned from 0 to $1200 \mathrm{~mW}$. Another laser is a narrow bandwidth fiber laser (NFL) which is amplified by the EDFA, and injected into the sensing fiber through the polarization scrambler and the WDM. The backward signal passes through the fiber splitter, the narrow bandwidth reflection filter, and the WDM. The signal of SBS frequency shifts is acquired by detecting the beat frequency, which is caused by coherently detecting the SBS backscattering signal through $\mathrm{C} 1$ and the local NFL laser signal. Finally, it is sent to the $\mathrm{PC}$ control center for further analysis to achieve the strain information along the fiber. This sensor system has been granted a China Invention Patent.

\subsection{Distributed optical fiber Brillouin photon sensors integrated with a fiber Brillouin frequency shifter [36]}

The sensor integrates a fiber Brillouin frequency shifter and a distributed optical fiber Brillouin photons sensor, which is based on the frequency shifting effect of fiber Brillouin scattering, the broadband nonlinear amplifying effect of the optical fiber, strain and temperature sensitive effects of Brillouin scattering of coherent amplifying, and the optical time domain reflection principle. It contains a narrow linewidth fiber laser, three fiber splitters, a pulse modulator, a Brillouin frequency shifter, two fiber circulators, an EDFA, a polarization scrambler, a narrow bandwidth reflection filter, a Raman pump laser, single mode sensing fibers, a fiber filter, a photo-electricity receiver and an amplifying module, two digital signal processors, a photo-electricity heterodyne receiving and amplifying module, and a computer. The Brillouin frequency shifter is connected with a circulator, a single fiber, and an F-P filter. This sensor has advantages of the simple structure, low cost, high measuring accuracy and good stability. Z. Zhang team proposed a novel BOTDA and solved the difficulty which needed to lock the frequency difference of the narrow bandwidth probing laser and the pumping laser in the BOTDA system invented by T. Horiguchi. In order to improve the measuring accuracy of the BOTDA and BOTDR, the researchers $[33,36]$ used the schemes to reduce the local laser signal $\left(v_{0}\right)$ and realize the coherent detecting through the microwave generator, electro-optics or acousto-optic modulator, and microwave shifter. It is very difficult and expensive to detect microwave $(11 \mathrm{GHz})$ signal if using heterodyne coherent detection between Brillouin backscattering signal $\left(v_{0}-v_{\mathrm{B}}{ }^{\prime}\right)$ with the strain and temperature information and the local laser signal $\left(v_{0}\right)$ at the range of several $\mathrm{GHz}$ because the frequency of Brillouin phonon is about $11 \mathrm{GHz}$.

The setup of the distributed optical fiber Brillouin photons sensor based on the fiber Brillouin frequency shifter is shown in Fig. 13. It contains a narrow linewidth single frequency fiber laser, three fiber splitters, a pulse modulator, a Brillouin frequency shifter (BFS), two circulators, an EDFA, a polarization scrambler (PS), two fiber filters, a fiber Raman pump laser, single mode sensing fibers, a photo-electric receiver module and an amplifying module (M1), a photo-electricity heterodyne receiver module and an amplifying module (M2), two DSPs, and a computer. The Brillouin frequency 
shifter is connected with a circulator, single mode fibers, and an optical fiber F-P filter. Filterl is a narrow bandwidth reflection fiber filter, and Filter2 is a fiber filter.

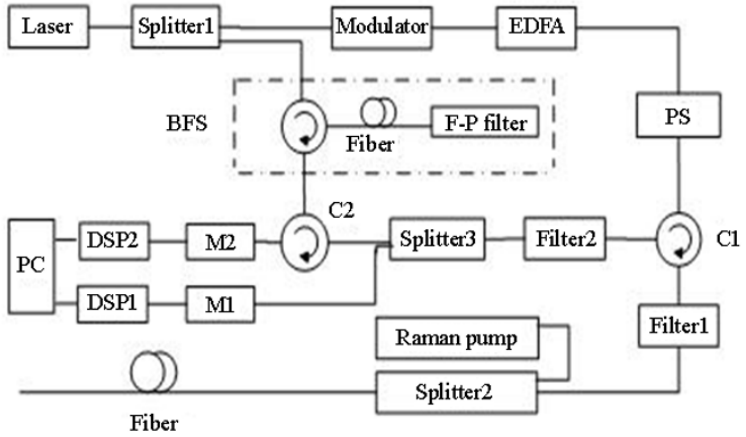

Fig. 13 Distributed fiber Brillouin photon sensor integrated with a fiber Brillouin shifter.

In this system, a narrow linewidth single frequency fiber laser is used as the sensing light source. The laser light is modulated by the pulse modulator through Splitter1 and is then amplified by the EDFA. The amplified pulse passes through a PS and enters into a narrow bandwidth reflection fiber filter through the circulator C1. A fiber Raman pump laser is employed as the pump source which is injected into the single mode sensing fiber while the laser pulse passes through Splitter2. The fiber Raman pump laser and the single mode sensing fiber construct the pump fiber Raman amplifier. The backscattering signal passes through Filterl to reduce noises, which are separated into two parts by Splitter3 through $\mathrm{C} 1$. One part is injected into M1 as well as the other part is injected into M2 through C2.

The laser light via Splitter1 is injected into the BFS and then passes through $\mathrm{C} 2$. The signal caused by the Brillouin frequency shifter is reflected by Filter2 via Splitter2 and then passes through M2. Both the signal from the BSF and Brillouin backscattering signal are processed by DSP2. Finally, all the signals through DSP1 and DSP2 are sent to the computer for the analysis. As a result, a distributed optical fiber Brillouin sensor integrated with the fiber Brillouin frequency shifter can be realized, which has many advantages, such as simple structure, low cost, high measuring accuracy, and good stability.

\section{Industrialization and application of the distributed optical fiber Raman scattering photon sensor}

Exploring new generation optic fiber sensing mechanism based on nonlinear optics effects is our work, which can improve the spatial resolution, temperature measurement precision, measuring length, measuring time and improving the system reliability of the distributed optical fiber sensor system. According to the requirement of different applications, realizing multi-parameter test is the key for current sensing technologies. The Internet of things combined with the optical fiber sensing nets and Internet or wireless network is the developing trend. Sensing nets are important parts of the Internet of things, which is a new technology revolution in this century. The strategies of "smart earth" and "sensing China" can bring both short-term and long-term good benefits and will drive to a billionaire market.

The research team at China Jiliang University is one of the earliest research teams to engage in the study of distributed optical fiber Raman temperature sensors. Two patents named "distributed optical fiber Raman photon sensors fire detection" and "online real-time fiber grating fire monitoring system" were granted successfully in 2010 and 2011. In order to promote the industrialization of distributed optical fiber sensors, we combined with Weihai Beiyang Electric Joint Group, and established CJLU-Weihai Beiyang Electric Joint Group Optical Fiber Sensing Research Center and Hangzhou OE Tech. Co, Ltd.

\subsection{Industrialization of the distributed optical fiber Raman scattering photon sensor [22]}

At the end of 1980s, the YORK Company, established by University of Southampton, started to produce DTS type of distributed optical fiber Raman 
temperature sensors. Later, more companies produced various types of distributed optical fiber Raman temperature sensors, which were widely applied to large civil constructions, transportations, tunnels, dams, power engineering, oil chemical industry, coal mine engineering and became an important mean of industrial on-line monitoring in the late 1990s and early 2000s.

In 2006, in order to realize the industrialization of existing research results, the CJLU team established Hangzhou OE Tech. Co. Ltd. in Hangzhou National University Science and Technology Park. Two years later, the team cooperated with Weihai Beiyang Electric Group, began the mass production of short-range, medium-range, long-range distributed optical fiber Raman temperature sensors in large quantities and formed a series of products, which were FGWC type S-02, 04 short-range distributed optical fiber Raman temperature sensor series, FGWC type M-08, 10, 12, 15 medium-range distributed optical fiber Raman temperature sensor series, and FGWC type L-30 long-range distributed optical fiber Raman temperature sensor series [23], which are shown in Table 2. The characteristic comparison of the distributed optical fiber Raman photon temperature sensor system with other products are shown in Tables 2, 3, 4 and 5 .

Table 2 Characteristics of distributed fiber Raman temperature sensors at Hangzhou OE.

\begin{tabular}{lccc}
\hline & FGWC-S02, S04 & FGWC-M08,10, 12, 15 & FGWC-L30 \\
\hline Fiber length $(\mathrm{km})$ & 2,4 & $8,10,12,15$ & 30 \\
Temperature accuracy $\left({ }^{\circ} \mathrm{C}\right)$ & \pm 1 & \pm 1 & \pm 2 \\
Temperature resolution $\left({ }^{\circ} \mathrm{C}\right)$ & 0.1 & 0.05 & 0.1 \\
Spatial resolution $(\mathrm{m})$ & 1.5 & 1.5 & $<4$ \\
Sample resolution $(\mathrm{m})$ & 1 & 1 & 1 \\
Measurement time $(\mathrm{s})$ & 5,10 & 10 & $<7$ \\
\hline
\end{tabular}

Table 3 Characteristics of short distance distributed fiber Raman temperature sensors.

\begin{tabular}{lccc}
\hline & DTS300 (Sensa) & YOKOGAWA & FGWC-S02,S04 (Hangzhou OE) \\
\hline Fiber length $(\mathrm{km})$ & 2 & 2 & 2,4 \\
Temperature accuracy $\left({ }^{\circ} \mathrm{C}\right)$ & \pm 1 & \pm 1 & \pm 1 \\
Temperature resolution $\left({ }^{\circ} \mathrm{C}\right)$ & 0.1 & 0.1 & 0.1 \\
Spatial resolution $(\mathrm{m})$ & 3 & 4 & 1.5 \\
Sample resolution $(\mathrm{m})$ & 2.5 & 2 & 1 \\
Measurement time $(\mathrm{s})$ & 20 & 30 & 5,10 \\
\hline
\end{tabular}

Table 4 Characteristics of middle distance distributed fiber Raman temperature sensors.

\begin{tabular}{lcccc}
\hline & N4385A/N4386A (Agilent) DTS800M10 (Sensa) DTS5100 (Sensortran) & FGWC-M (Hangzhou OE) \\
\hline Fiber length $(\mathrm{km})$ & $8(\max )$ & 10 & 10 & $8,10,12,15$ \\
Temperature accuracy $\left({ }^{\circ} \mathrm{C}\right)$ & \pm 2 & \pm 2 & 0.1 & \pm 1 \\
Temperature resolution $\left({ }^{\circ} \mathrm{C}\right)$ & 0.1 & 0.1 & 1 & 1.5 \\
Spatial resolution $(\mathrm{m})$ & 1.5 & 1. & 1 & 1 \\
Sample resolution $(\mathrm{m})$ & 1 & 1 & 30 & 10 \\
Measurement time $(\mathrm{s})$ & 60 & 600 & &
\end{tabular}


Table 5 Characteristics of long distance distributed fiber Raman temperature sensors.

\begin{tabular}{lccc}
\hline & DTS800S30(Sensa) & DTS-XR(Sentinel) & FGWC-L30(Hangzhou OE) \\
\hline Fiber length $(\mathrm{km})$ & 30 & 30 & 30 \\
Temperature accuracy $\left({ }^{\circ} \mathrm{C}\right)$ & \pm 2 & \pm 1.2 & \pm 2 \\
Temperature resolution $\left({ }^{\circ} \mathrm{C}\right)$ & 0.1 & 0.05 & 0.1 \\
Spatial resolution $(\mathrm{m})$ & 8 & 2 & $<4$ \\
Sample resolution $(\mathrm{m})$ & 2 & 1 & 1 \\
Measurement time $(\mathrm{min})$ & 10 & 1 & $<7$ \\
\hline
\end{tabular}

\subsection{Application of the distributed optical fiber Raman scattering photon sensor [37-39]}

Cooperating successively with Weihai Beiyang Electric Joint Group, Shanghai OE Power Technology Co. Ltd., and Hangzhou OE Tech. Co. Ltd., China Jiliang University developed a lot of applied research works and completed a lot of projects such as: the optical fiber temperature sensor network engineering of Shandong Rizhao port, the $110-\mathrm{kV}$ substations of ultrahigh Mengzi underground fiber-optic temperature sensor control and detection network project of Shanghai World Expo project, the Shanghai Tibet south road tunnel engineering optical fiber temperature monitoring network of Shanghai World Expo project, the Shanghai Yangtze river tunnel fiber temperature sensing nets, the Shanghai Donghai bridge wind power optical fiber temperature sensor network engineering, the Shanghai Caojing power plant fuel conveying corridor and circular indoor temperature sensing coal storage fiber nets, the Shanghai east Fuxing road tunnel and cable corridor of the fire monitor by the optical fiber fire monitoring network engineering, and the Xiaowan hydropower station optical fiber sensing nets engineering. Two China invention patents named "distributed fiber Raman photon sensing temperature fire detector" and "on line, real time fiber grating fire detection system" were granted [37, 38], and the accumulative total output value was more than 500 million RMB. Some examples are listed as below.

China Jiliang University and Weihai Beiyang
Electric Joint Group undertook many engineering projects and successfully developed the BY-DTS distributed optical fiber temperature measuring and warning system which could be used in the electric power industry, mainly used in high voltage power cables and cable joint temperature and fault points, cable shafts, bridges, interlinings and in cable trench temperature and cable tunnels fire behavior. The system structure is shown in Fig. 14. By installing the sensor cable in main cables, the cable connector and cable interlinings, shafts, bridges, the system formed the power system fire monitoring fiber temperature sensing nets.

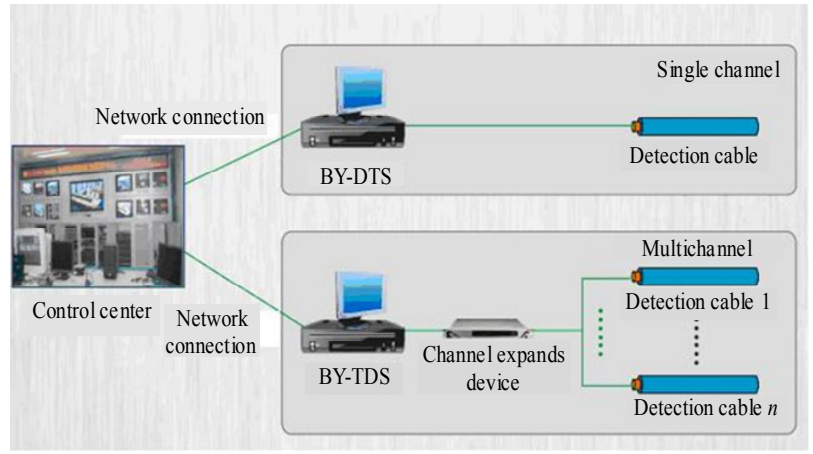

Fig. 14 Structure of the BY-DTS distributed fiber fire early alarming system.

China Jiliang University and Weihai Beiyang Electric Joint Group undertook the port belt-conveyor fire warning optical fiber sensing network of Shandong Rizhao port. In the port and dock, belt transmission lines are the basic transportation. The belt out of order will rub with rollers and produce an extremely large amount of heat during transmissions, which will lead to fire. The distributed optical fiber temperature measuring 
warning system can monitor important equipments, such as rollers by temperature on-line monitoring and long artificially inspection cycle, large strength, the no timely response. It has been hot so clear and timely at an early warning, to ensure the operational efficiency of the loading and unloading equipment in the port, and reduce or avoid accident injuries and property losses. The main monitoring object is the belt-conveyor fire warning system, roller, and roller temperature monitor. The system structure is shown in Fig. 15.

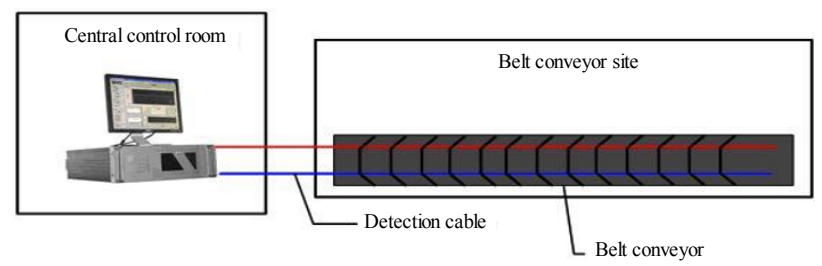

Fig. 15 Structure of the fire early alarming fiber sensor system.

China Jiliang University and Shanghai OE Power Technology Co. Ltd. undertook a project of Shanghai World Expo related to the monitoring network of the optical fiber sensing net engineering, which basically was the temperature monitoring of the power cables, power generation, transmission equipments, power cables of the temperature monitoring and carry traffic on the preliminary analysis. At the same time, it contributed to the security of the World Expo in Shanghai electric power supply and the fire monitoring. The $110-\mathrm{kV}$ ultrahigh voltage Mengzi underground substation monitoring of distributed optical fiber Raman temperature sensing nets is shown in Fig. 16. The mainframe of the distributed fiber Raman temperature sensor system is shown in Fig. 17.

China Jiliang University and Shanghai $\mathrm{OE}$ Power Technology Co. Ltd. undertook the project "Shanghai Yangtze river's bridge engineering cable tunnel monitoring temperature and fire alarm distributed optical fiber Raman temperature sensor network engineering", which is shown in Fig.17. Shanghai Yangtze river bridge engineering channel tunnel up in the south Pudong fifth ditch connects Shanghai suburb ring, across the Yangtze river in south port waters, Changxing island, Yangtze river north port water area and stops at Chenjia town, Chongming island. One way to the tunnel through the Yangtze river in the south port water area is about $8.9 \mathrm{~km}$ long, and the way to bridge across the Yangtze river north port water area is about $10.3 \mathrm{~km}$ long; the wiring road of Changxing island and Chongming island is about $6.3 \mathrm{~km}$ long, and the total investment of the project is about 126 million yuan, which is shown in Fig. 18.

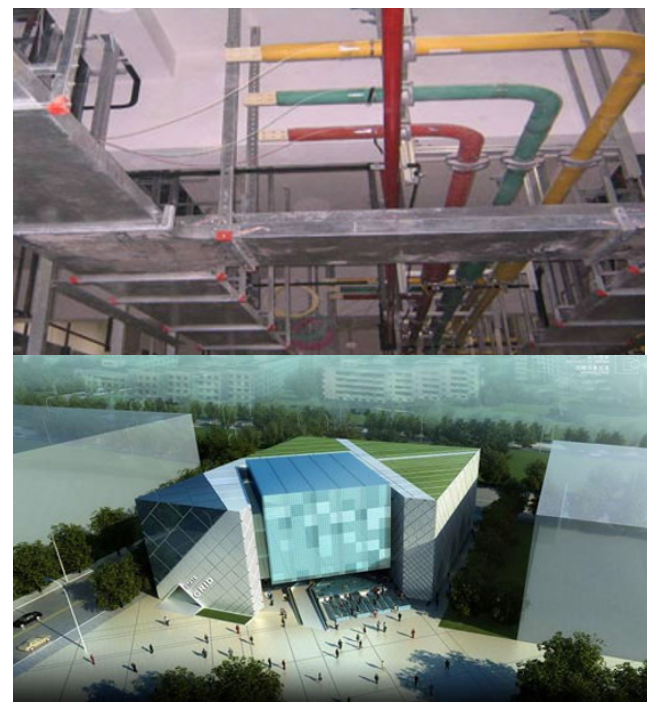

Fig. 16 Setup of the $110-\mathrm{kV}$ ultrahigh voltage Mengzi underground transformer substation of Shanghai World Expo's fair.

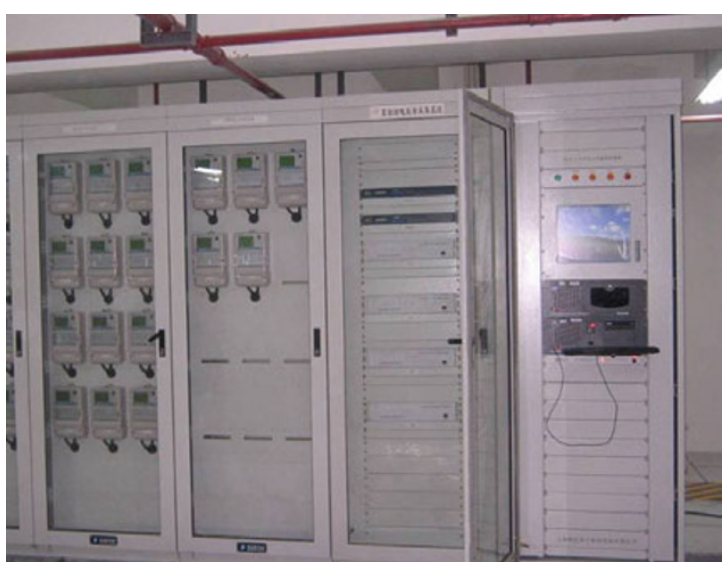

Fig. 17 Mainframe of a distributed fiber Raman photon temperature sensor system. 


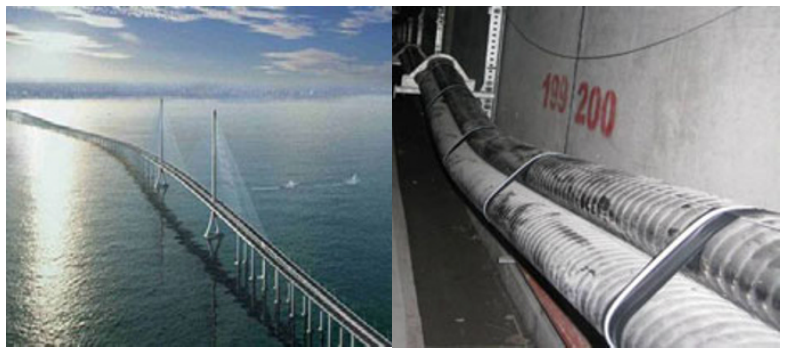

Fig. 18 Shanghai Yangtze River tunnel bridge by using optical fiber Raman temperature sensing networks.

\section{Conclusions}

The new generation of the fiber sensor mechanism based on optical fiber nonlinear scattering fusion effect has been discussed in this paper. The keys of recent developed optical sensor technologies are longer measurement distance, improved measurement accuracy, space resolution, reliability of the system and multi-parameter measurments. The amplification effect of Rayleigh and Brillouin scattering in the optical fiber has been researched, and new generation of the optical fiber sensor technology based on the optical fiber nonlinear scattering fusion principle has been proposed, which uses various nonlinear scattering effects in optical fibers. A series of new generations of full distributed optical fiber sensor technologies are studied and designed, which consist of: a new generation of the ultra-long distance full distributed fiber Raman and Rayleigh scattering photon sensors integrated with a fiber Raman amplifier; self-correct full distributed fiber Raman photon temperature sensors based on Raman correlation dual sources; full distributed fiber Raman photon temperature sensors based on the pulse code source and technology; full distributed fiber Raman photon temperature sensors integrated with a fiber Raman shifter; a new type of the BOTDA integrated with a fiber Raman amplifier for replacing the fiber Brillouin amplifier; full distributed fiber Raman and Brillouin photon sensors integrated with a fiber Raman amplifier; full distributed fiber Brillouin photon sensors integrated with a fiber Brillouin shifter.

The Internet of things composed of sensor networks is a developing direction. The full distributed fiber Raman Rayleigh and Brillouin photon sensor is a $3 \mathrm{~S}$ (smart materials, smart structure, and smart skill) system and consists of a new generation of the intellect fiber sensor network. The star and annular local area sensor network is used by the time division multiplexer (TDM) and WDM technology, and the global intellect sensor network is composed of the local area sensor network through the wireless network and Internet.

\section{Acknowledgment}

This work is supported by the National Basic Research Program of China (973 Program) under Grant No. 2010CB327804.

Open Access This article is distributed under the terms of the Creative Commons Attribution License which permits any use, distribution, and reproduction in any medium, provided the original author(s) and source are credited.

\section{References}

[1] Z. Zhang, I. S. Kim, J. Wang, H. Feng, N. Guo, and Y. Kim, "Distributed optical fiber sensors system and networks," in Proc. SPIE, vol. 4357, pp. 35-53, 2000.

[2] Z. Zhang, T. Liu, and B. Zhang, "Laser Raman type distributed optical fiber temperature sensor system," Acta Optica Sinica, vol. 15, no. 11, pp. 1586-1589, 1995 (In Chinese).

[3] T. Liu and Z. Zhang, "Optical fiber backward Raman scattering temperature character and it's application," Chinese Journal of Lasers, vol. 22, no. 4, pp. 250-252, 1995 (In Chinese).

[4] Z. Zhang, J. Wang, H. Feng, N. Guo, X, Yu, X. Wu, I. S. Kim, and Y. Kim, "Measurement of performance characters of a $10 \mathrm{~km} \mathrm{LD} \mathrm{distributed} \mathrm{optical} \mathrm{fiber}$ temperature sensor (LDOFTS) system," in Proc. SPIE, vol. 4220, pp. 245-249, 2000.

[5] Z. Zhang, N. Guo, X. Yu, J. Wang, X. Wu, I. S. Kim, and Y. Kim, "Distributed fiber optics Raman high temperature $\left(1000{ }^{\circ} \mathrm{C}\right)$ measuring networks," in Proc. 
SPIE, vol. 4603, pp. 116-120, 2001.

[6] Z. Zhang, H. Liu, N. Guo, X. Wu, X, Yu, H. Feng, and I. S. Kim, "The optimum designs of $30 \mathrm{~km}$ distributed optical fiber Raman photons temperature sensors and measurement network," in Proc. SPIE, vol. 4920, pp. 268-279, 2002.

[7] Z. Zhang, H. Liu, and J. Wang, "Optimum design of $30 \mathrm{~km}$ long-distance distributed optical fiber Raman temperature sensor system (invited paper)," in Proc. SPIE, vol. 5634, pp. 182-190, 2004.

[8] Z. Zhang, B. Dai, L. Li, H. Xu, H. Liu, J. Wang, C. Li, and I. S. Kim, "Amplification effect on stimulated Brillouin scattering in the S-band forward G652 fiber Raman amplifier," Chinese Optics Letters, vol. 3, no. 11, pp. 629-632, 2005.

[9] Z. Zhang, B. Dai, L. Li, et al., "Experimental study of Raman amplification on stimulated brillouin scattering in the G652 fibers at $1520 \mathrm{~nm}$," in Proc. SPIE (the 20th Congress of the International Commission for Optics (ICO 20)), vol. 6027, pp. 6027-6042, 2006.

[10] G. P. Agrawal, Nonlinear fiber optics and applications of nonlinear fiber optics. New York: Academic Press, 2008.

[11] Z. Zhang, "The research of temperature effect of optical fiber molecular and application progress for distributed optical fiber temperature sensor network," Journal Atomic and Molecular Physics, vol. 17, no. 3, pp. 559-565, 2000 (In Chinese).

[12] Z. Zhang, S. Jin, and J. Wang, "The research of distributed optical fiber Raman gain amplifier," in Proc. SPIE, vol. 4579, pp. 54-56, 2001.

[13] R. H. Stolen and E. P. Ippen, "Raman gain in glass optical waveguides," Applied Physics Letters, vol. 22, no. 6, pp. 276-278, 1973.

[14] T. Yang, X. Yin, W. He, and C. Fu, "Gain spectrum of Raman fiber amplifier," Acta Optica Sinica, vol. 22, no. 11, pp. 1335-1339, 2002 (In Chinese).

[15] Z. Zhang, B. Dai, J. Wang, H. Liu, H. Xu, D. Geng, L. Li, C. Li, and I. S. Kim, "Gain flattened distributed fiber Raman amplifier," Optoelectronics Letters, vol. 3, no. 5, pp. 339-341, 2007.

[16] B. Dai, Z. Zhang, and C. Li, "Design of S-band gain flattened distributed fiber Raman amplifier with chirped fiber Bragg grating filter," Optoelectronics Letters, vol. 2, no. 1, pp. 9-11, 2006 (In Chinese).

[17] B. Dai, Z. Zhang, and G. Dan, "Study and manufacture of gain flattened S-band distributed dispersion compensation fiber Raman amplifier," in Proc. SPIE (the 20th Congress of the International Commission for Optics (ICO 20)), vol. 6027, pp. 602701, 2006.

[18] B. Dai, Z. Zhang, Y. Huang, C. Li, Y. Huang, H. Liu, L. Li, D. Geng, T. Liu, and I. S. Kim, "Manufacture and test of gain flattened C-band and S-band distributed dispersion compensation fiber Raman amplifier," in Proc. SPIE (Asia Pacific Optical Communication (APOC) 2005), vol. 6019, pp. 601920, 2005.

[19] Z. Zhang, L. Li, and D. Geng, "Study on forward Stimulated Brillouin scattering in a backward pumped fiber Raman amplifier," Chinese Optics Letters, vol. 2, no. 11, pp. 627-629, 2004.

[20] Z. Zhang, L. Li, and D. Geng, "Forward cascaded Stimulated Brillouin scattering in an S-band distributed G652 fiber Raman amplifier (invited paper)," in Proc. SPIE (Photonics in ASIA 2004), vol. 5633, pp. 110-119, 2005.

[21] Z. Zhang and H. Gong, "Amplification effect on SBS and Rayleigh scattering in the backward pumped distributed fiber Raman amplifier," Chinese Optics Letters, vol. 7, no. 5, pp. 393-395, 2009.

[22] Z. Zhang, S. Jin, J. Wang, H. Liu, Z. Sun, H. Gong, $\mathrm{X}$. Yu, and W. Zhang, "Distributed optical fiber Raman photon sensor research review," Chinese Journal of Lasers, vol. 37, no. 11, pp. 2749-2761, 2010 (In Chinese).

[23] Z. Zhang and J. Wang, "Ultra-long distance distributed optical fiber Raman photon temperature sensor integrated a Raman amplifier," China Utility Model Patent, 20072019219.X, Aug. 27, 2008.

[24] Z. Zhang, "Full distributed Rayleigh and Raman scattering photons stress and temperature sensor," China Invention Patent, 200910099463.7, Jun. 8, 2009.

[25] Z. Zhang, X. Yu, J. Wang, and S. Jin, "A pulse encode full distributed fiber Rayleigh and Raman scattering photon sensor," China Utility Model Patent, 201120287620.X, Dec. 20, 2011.

[26] Z. Zhang, "Auto-correction distributed fiber Raman temperature sensor based on Raman-relation dual wavelength source," China Invention Patent, 20091012201.1, Jan. 5, 2011.

[27] Z. Zhang, J. Wang, W. Zhang, X. Yu, and X. Niu, “A dispersion and loss spectra of a new type auto-correction distributed optical fiber Raman temperature sensor," China Utility Model Patent, 20102015461.7, Nov. 17, 2011.

[28] Z. Zhang, J. Wang, and S. Jin, "A Raman-relation dual wavelength source distributed fiber Raman temperature sensor fused the fiber Raman shifter," China Utility Model Patent, 201020634363.8, Jun. 29, 2011.

[29] X. Yu, Z. Zhang, and W. Zhang, "Distributed optical fiber Raman temperature sensor used serious pulse encode and decode optical source," China Invention Patent, 201010169596.X, Aug. 17, 2011. 
[30] Z. Zhang, S. Li, J. Kong, J. Wang, and S. Jin, “An ultra-long distance full distributed fiber Rayleigh and Raman scattering photon sensor fused fiber Raman shifter," China Utility Model Patent, 201020634344.5, Jun. 29, 2011.

[31] Z. Zhang, S. Li, J. Kong, J. Wang, and S. Jin, “An ultra-long pulse code full distributed fiber Rayleigh and Raman scattering photon sensor fused fiber Raman shifter," China Utility Model Patent, 201120286494.6, Dec. 22, 2011.

[32] Z. Zhang and S. Zhang, "New type optical fiber Brillouin optics domain analyzer (BOTDA)," China Invention Patent, 200810063711.8, Jul. 25, 2010.

[33] Z. Zhang, S. Jin, J. Wang, H. Gong, and Y. Li, "A distributed optical fiber Raman, Brillouin scattering sensors," China Utility Model Patent, 201020157449.6, Dec. 12, 2010.

[34] Z. Zhang, "Ultra-long distributed optical fiber Raman, Brillouin scattering sensors," China Invention Patent, 200710156868.0, May 27, 2009.

[35] G. Bolognini, M. A. Soto, and F. Di Pasquale,
"Fiber-optic distributed sensor based on hybrid Raman and Brillouin scattering employing multiwavelength Fabry-Pérot lasers," IEEE Photonics Technology Letters, vol. 21 no. 20, pp. 1523-1525, 2009.

[36] Z. Zhang, H. Gong, Y. Li, and S. Jin, "An distributed fiber Brillouin sensor fused fiber Brillouin shifter," China Utility Model Patent, 201020634326.7, Nov. 26, 2010.

[37] Z. Zhang and W. Zhang, "Distributed fiber Raman photon sensing temperature fire detector," China Invention Patent, 200810120192.4, Jul. 27, 2011.

[38]Z. Zhang, X. Dong, H. Gong, and S. Zhang, "On line, real time fiber grating fire detector system," China Invention Patent, 200810120191.X, May. 4, 2011.

[39] H. Wu, "Distributed fiber Raman temperature sensor applied to Shanghai smart power grid," presented at the 5th forum on sensor develop and industrialization, Guangzhou, China, Jun. 30-Jul. 2, 2010 (In Chinese). 